\title{
PINWORM CONTROL AND RISK FACTORS OF PINWORM INFECTION AMONG PRIMARY-SCHOOL CHILDREN IN TAIWAN
}

\author{
JOHN F. C. SUNG, RUEY S. LIN, KUO C. HUANG, SIN Y. WANG, AND YUN-JU LU \\ Institute of Environmental Health and Institute of Epidemiology, National Taiwan University College of Public Health, \\ Taipei, Taiwan
}

\begin{abstract}
Longitudinal pinworm (Enterobius vermicularis) infection rates were estimated at a mass screening for first-grade children during 1991-1996; children were provided medication at the screening. This campaign was able to decrease the infection rates for the 1991 cohort from $16.3 \%$ to $0.6 \%$. A case-control study was further conducted for the investigation of risk factors among fourth-graders. Cases comprised 429 children with at least one infection between September 1996 to June 1999, and controls were 280 randomly selected uninfected classmates. Parents were asked to complete a questionnaire survey to report students' personnel hygiene habits. The case-control study revealed that significant factors associated with the infection included playing on the floor (odds ratio [OR], 2.5), nail biting (OR, 2.1), failure to wash hands before meals (OR, 1.7) and living in nonapartment dwellings (OR, 1.6). Girls were at a higher, but not significant, risk (OR, 1.4), than boys. In conclusion, inadequate personal hygiene increases the risk for pinworm infection. The mass screening-medication campaign can be adapted to countries with a similar parasitic problem.
\end{abstract}

\section{INTRODUCTION}

Parasitic infections among children remain a public health concern in developing regions. Even in the 1990s, the infection rate for intestinal nematodes is as high as $98 \%$ in some villages in underdeveloped areas. ${ }^{1,2}$ Taiwan was once an area endemic for a high prevalence of nematode intestinal parasites among children. The helminth infection rate among primary-school-age children in 1971 was as high as $73 \%$ - and 55\% for infection with Ascaris lumbricoides alone. ${ }^{3}$ The Taiwan Provincial Health Department initiated a 4-year plan to control intestinal helminths, with a overall infection rate falling to $13.7 \%$ in 1975 . They established a Parasite Control Association (PCA) to continue 2 additional 5-year plans aiming for more thorough control of intestinal helminths. At the end of these plans, in 1986, the overall infection rates decreased to $0.19 \%$. The parasite control and prevention program then concentrated on pinworms (Enterobius vermicularis) for primary-school children, except for several remote mountain areas, where the screening-medication measure for Ascaris was still in place.

Pinworm screening data provided by the PCA suggest an apparent parasite reinfection phenomenon. ${ }^{3}$ There are few cross-sectional studies investigating the factors associated with pinworm infection. ${ }^{4-7}$ This report describes a case-control study to investigate the factors associated with the recent infection of pinworms among fourth-graders in selected primary schools. The infection pattern by the mass screeningmedication campaign against pinworm infection is described briefly as well.

\section{MATERIALS AND METHODS}

Taiwan is an island nation located east of China between Okinawa and the Philippines. It has a population of $\sim 21.8$ million in 1998 , living in an area nearly $36,000 \mathrm{~km}^{2}$, or 13,900 square miles, ${ }^{8}$ in size.

Trend analysis for mass screening. With the support from and supervision of the Provincial Health Department, County and City Health Bureaus, the Provincial Education Department, and County/City Education Bureaus, the PCA organized the pinworm control program for primary-school students. The PCA provided supplies and personnel training and parasitology laboratories. Parasite eggs were screened by $5-15$ trained medical technologists assigned to each laboratory on the basis of the size of the student body in each of the 21 counties and cities in Taiwan. The PCA technicians collected the microscopically examined specimens for $>2$ million primary-school students. An adhesive thin cellophane tape swab method was used for screening the perianal region for pinworm eggs. Four screening examinations were conducted each school year for all primary-school students in Taiwan Province. In the Kaohsiung metropolitan area, instead of being sampled 4 times, students were only sampled twice annually, once in spring and the other in fall.

Each year in Taiwan Province, the first screening activity began in early September for half of the fall semester, and the second screening was conducted in the second half of fall semester, ending in January. The third screening began in February for half of the spring semester, and the fourth screening was conducted in the rest of the spring semester, ending in late June. A 10 -week summer break followed. Students who tested positive and their family members living in the same houses received medication (one 10-mg mebendazole pill). Public health nurses at local health departments and teachers at all involved schools assisted in distributing the medicine. For students living in remote mountain areas, the screening-medication campaign for Ascaris and other nematode intestinal parasites remained in place because of higher prevalence rates. Teachers also conducted health education, advising students of personal hygiene for parasite prevention. Students' parents were asked to pay a small fee for screening examinations and medication, although lowincome families were exempt.

Data for analyzing pinworm infection prevalence rates were taken from the public databases generated by the PCA. This report displayed the chronological prevalence of pinworm infection among students begun with their first grade, by year admitted to school, between 1991 and 1995. They were followed to the fourth screening in 1996. The results demonstrated a reinfection pattern deserving attention. 


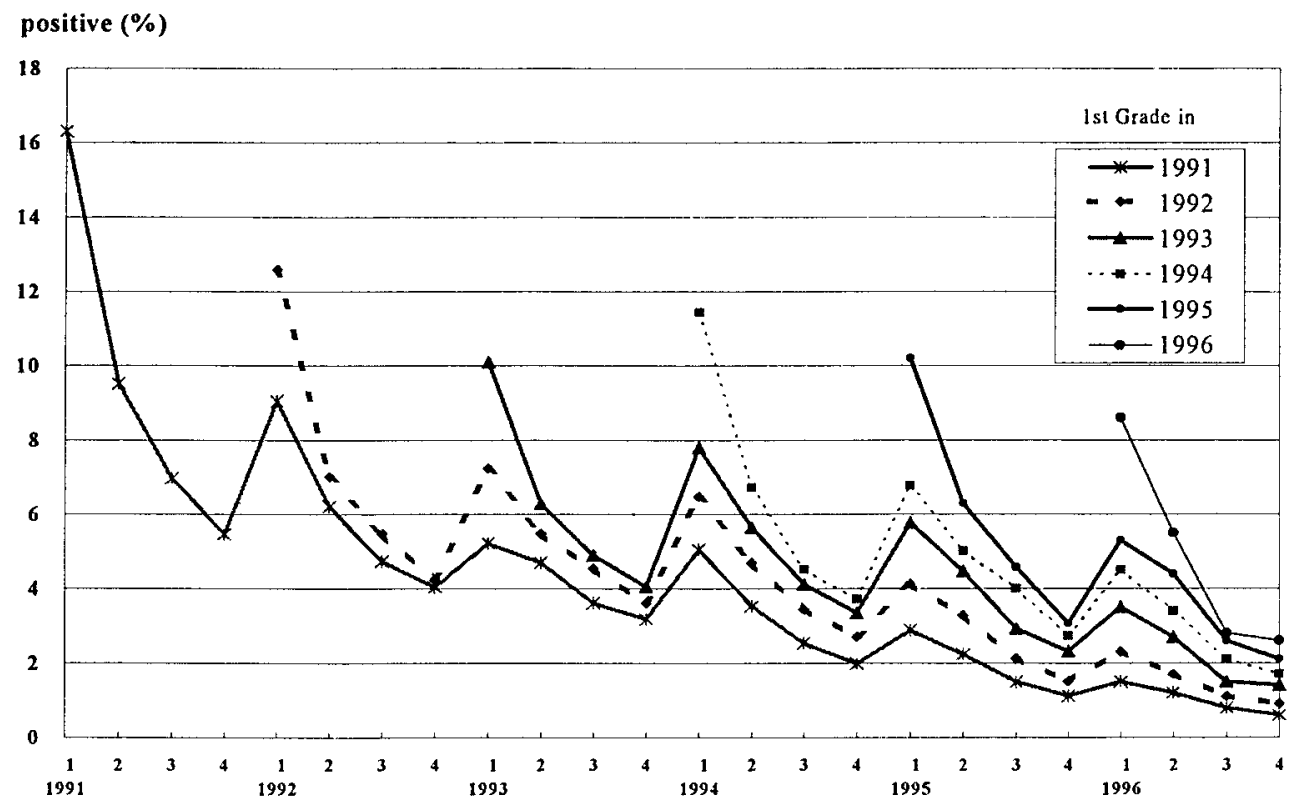

Screening sequence and year

FIGURE 1. Percentage of children with a positive finding of pinworm eggs by use of the sequence $(1,2,3,4)$ of screening examinations for cohorts beginning with the first-graders admitted to schools in 1991, 1992, 1993, 1994, 1995, and 1996. $1=$ first screening in a school year covering the first half of the fall semester; $2=$ second screening covering the second half of the fall semester; $3=$ third screening covering the first half of the spring semester; $4=$ fourth screening covering the second half of the spring semester.

Sampling and data analysis of case-control study. A case-control study on risk factors associated with pinworm infection was conducted among fourth-graders of 15 randomly selected elementary schools in Taichun County, representing rural townships, and in metropolitan Kaohsiung, representing urban cities. Pinworm egg screening records available between September 1996 and January 1999 for children in these 15 schools were reviewed and 280 students, all with negative results and 429 students with at least one positive swab examination, were randomly selected from these 15 schools. A questionnaire was given between January and April 1999 to each student's parents, asking about their child's personal hygiene habits. The questionnaire for parents to complete was reviewed and approved by a committee consisting of officers from the Provincial Department of Health, representatives from PCA, and university faculty. With parental consent, the information we collected included medication, leisure, fingernail-biting, and hand-washing behaviors; household members; number of people sharing the bedroom with the student; type of bed; type of house; and frequency of bathing and change of underclothes.

For the case-control study on factors associated with pinworm infection, the data were analyzed first by a frequency distribution method to review the distribution of responses and risk factors to estimate differences between case patients and controls. Variables with potential confounding effect were used for logistic regression analyses to calculate the strength of the association between infection and risk factors by odds ratio (OR) and $95 \%$ confidence interval (CI). Crude ORs were estimated by univariate analyses, and adjusted ORs were then estimated by multivariate logistic regression analysis, which included all variables significant at $0.10 \mathrm{lev}-$ el in the univariate analyses. A greater OR indicates an el- evated risk. Data were analyzed by SAS for Windows version 6.12 (SAS Institute, Cary, NC).

\section{RESULTS}

Trend of pinworm control. The public-use data generated by the PCA from 1991 to 1996 were used to demonstrate the effectiveness of pinworm control in recent years. Figure 1 shows changes in positive rates of pinworm egg screening for the cohorts of first-graders admitted to all primary schools in 1991, 1992, 1993, 1994, 1995, and 1996. The prevalence rate of pinworm infection was as high as $16.3 \%$ at the first screening for first-graders admitted in September 1991. Their infection rate decreased to $9.5 \%$ at the second screening and to $5.6 \%$ at the fourth screening of the school year in June 1992. After a summer break, the infection rate for this cohort rebounded to $9.1 \%$ at the first screening in the second school year, when they were second-graders in September 1992. Reinfection after the summer occurred for all other cohorts, all with the highest reinfection rate at the first screening of children in grade 2 . The reinfection rate decreased as the children grew and the screening-medication campaign continued. The infection rate for the 1991 cohort decreased to $0.6 \%$ at the fourth screening, for children in grade 6 , in 1996 . The infection rates for the later cohorts also decreased yearly.

Risk factors associated with the pinworm infection. Tables 1 and 2 show results from selected univariate analysis of factors that may be associated with infection for students in Taichung County and Kaohsiung City, respectively, in the case-control study. Pinworm control medication, playing on the floor, and fingernail-biting behaviors were significant risk factors for students with pinworm infection in both areas. 
TABLE 1

Comparison of personal hygiene and other factors between controls (0) and cases with positive pinworm egg findings in 1 and in $\geq 2$ screening examinations among fourth-graders, Taichung County, Taiwan

\begin{tabular}{|c|c|c|c|c|}
\hline \multirow[b]{2}{*}{ Variable } & \multicolumn{3}{|c|}{ No. of screenings positive for pinworm eggs, $n(\%)$} & \multirow[b]{2}{*}{$P$} \\
\hline & 0 & 1 & $\geq 2$ & \\
\hline \multicolumn{5}{|l|}{ Age (years) } \\
\hline Boys $\leq 9$ & $51(57.3)$ & $62(70.5)$ & $22(52.4)$ & 0.077 \\
\hline Boys $\geq 10$ & $38(42.7)$ & $26(29.6)$ & $20(47.6)$ & \\
\hline Girls $\leq 9$ & $32(47.1)$ & $26(51.0)$ & $21(72.4)$ & 0.066 \\
\hline Girls $\geq 10$ & $36(52.9)$ & $25(49.0)$ & $8(27.6)$ & \\
\hline \multicolumn{5}{|l|}{ Play on floor } \\
\hline Rare/No & $97(61.4)$ & $60(43.2)$ & $21(28.8)$ & 0.001 \\
\hline Sometimes & $54(34.2)$ & $65(46.8)$ & $40(54.8)$ & \\
\hline Always & $7(4.4)$ & $14(10.1)$ & $12(16.4)$ & \\
\hline \multicolumn{5}{|l|}{ Bite nails } \\
\hline No & 105 (66.9) & $71(50.7)$ & $35(48.6)$ & 0.011 \\
\hline Sometimes & $33(21.0)$ & $50(35.7)$ & $29(40.3)$ & \\
\hline Often & $19(12.1)$ & $19(13.6)$ & $8(11.1)$ & \\
\hline \multicolumn{5}{|l|}{ Wash hands } \\
\hline No & $8(5.1)$ & $11(7.9)$ & $5(6.9)$ & 0.032 \\
\hline Sometimes & $62(39.2)$ & $77(55.0)$ & $31(43.1)$ & \\
\hline Always & $88(55.7)$ & $52(37.1)$ & $36(50.0)$ & \\
\hline \multicolumn{5}{|l|}{ Family size } \\
\hline$\leq 5$ & $94(59.5)$ & $86(61.4)$ & $53(72.6)$ & 0.146 \\
\hline$\geq 6$ & $64(40.5)$ & $54(38.6)$ & $20(27.4)$ & \\
\hline \multicolumn{5}{|l|}{ Share bedroom with: } \\
\hline No one & $35(22.3)$ & $32(22.9)$ & $23(31.5)$ & 0.071 \\
\hline 1 person & $93(59.2)$ & $69(49.3)$ & $30(41.1)$ & \\
\hline$\geq 2$ people & $29(18.5)$ & $39(27.9)$ & $20(27.4)$ & \\
\hline \multicolumn{5}{|l|}{ Type of bed } \\
\hline Wood & $91(58.3)$ & $64(46.0)$ & $35(48.0)$ & 0.130 \\
\hline Tatame* & $6(3.9)$ & $8(5.8)$ & $7(9.6)$ & \\
\hline Simmons $\dagger$ & $59(37.8)$ & $67(48.2)$ & $31(42.5)$ & \\
\hline \multicolumn{5}{|l|}{ House style } \\
\hline Single-family detached & $27(17.2)$ & $25(17.9)$ & $17(23.3)$ & 0.565 \\
\hline Apartment & $17(10.8)$ & $11(7.9)$ & $4(5.5)$ & \\
\hline Townhouse & $113(72.0)$ & $104(74.3)$ & $52(71.2)$ & \\
\hline
\end{tabular}

Washing hands before meals among case patients was not as frequent as for controls. However, this was not significant for students in Kaohsiung.

Crude ORs obtained from univariate logistic regression analyses and adjusted ORs obtained from multivariate logistic regression analyses are shown in Table 3 . It shows that risk factors significantly associated with the pinworm infection were playing on the floor (OR, 2.52; 95\% CI, 1.80 3.51 ), nail biting (OR, 2.15; 95\% CI, 1.58-2.93), not washing hands before meals (OR, $1.71 ; 95 \% \mathrm{CI}, 1.23-2.37)$, and living in dwellings other than apartments (OR, 1.56; 95\% CI, 1.04-2.35). Those living in rural areas (Taichung County) were $33 \%$ less likely than those living in urban areas (Kaohsiung City) to have an infection or reinfection. Boys were at lower risk, but the difference was not significant.

\section{DISCUSSION}

Intestinal parasite disease prevention and control-an issue of lesser concern in most developed countries-is not considered in clinician's preventive services for children. However, intestinal nematode infection remains prevalent in many developing and underdeveloped countries. In Taiwan, the main campaign for prevention and control of helminthiasis was a prolonged project, lasting 14 years between 1972 and 1986 for primary-school children. ${ }^{3}$ In this time period, economic development positioned Taiwan into a higher economic status, with Taiwan soon classified as a developed area. ${ }^{9}$ The concurrent improvement in hygiene may have contributed to preventing parasite infection. We also believe that the campaign had an effective contribution, particularly in the first 4-year plan, in which blanket treatment prescriptions, the combination of santonin and kainic acid, were given to all students 4 times yearly because of the high infection rates. The infection rate subsequently declined rapidly by $59 \% .^{3}$

A greater effort thereafter was necessary to lower the infection rate to $0.19 \%$. This was achieved by giving medication to students with positive findings at the parasite screening. Although current costs for this campaign are much higher than 2 decades ago, the PCA considered it worthwhile to shift the effort against pinworm infection to all children islandwide.

It appears that it takes little time for children to become reinfected with Ascaris after receiving medication because it is a soil-transmitted parasite. However, helminthiases can be one of the causes of morbidity in underdeveloped areas, particularly for preschool children. Intestinal parasitism is one indicator of poor personal hygiene, lack of adequate environmental sanitation, overcrowding, and poverty. These factors must therefore be considered in developing a parasite prevention program. The Taiwan experience indicates that a 
TABLE 2

Comparison of personal hygiene and other factors between controls (0) and cases with positive pinworm egg findings in 1 and in $\geq 2$ screening examinations among fourth-graders, Kaohsiung Metropolitan, Taiwan

\begin{tabular}{|c|c|c|c|c|}
\hline \multirow[b]{2}{*}{ Variable } & \multicolumn{3}{|c|}{ No. of screenings positive for pinworm eggs, $n(\%)$} & \multirow[b]{2}{*}{$P$} \\
\hline & 0 & 1 & $\geq 2$ & \\
\hline \multicolumn{5}{|l|}{ Age (years) } \\
\hline Boys $\leq 9$ & $40(55.6)$ & $44(71.0)$ & $32(68.1)$ & 0.144 \\
\hline Boys $\geq 10$ & $32(44.4)$ & $18(29.0)$ & $15(31.9)$ & \\
\hline Girls $\leq 9$ & $34(64.2)$ & $33(62.3)$ & $40(78.4)$ & 0.156 \\
\hline Girls $\geq 10$ & $19(35.8)$ & $20(37.7)$ & $11(21.6)$ & \\
\hline \multicolumn{5}{|l|}{ Play on floor } \\
\hline Rare/No & $81(64.8)$ & $55(47.4)$ & $30(30.6)$ & 0.001 \\
\hline Sometimes & $38(30.4)$ & $47(40.5)$ & $55(56.1)$ & \\
\hline Always & $6(4.8)$ & $14(12.1)$ & $13(13.3)$ & \\
\hline \multicolumn{5}{|l|}{ Bite nails } \\
\hline No & $77(61.1)$ & $49(41.5)$ & $40(40.8)$ & 0.005 \\
\hline Sometimes & $38(30.2)$ & $48(40.7)$ & $37(37.8)$ & \\
\hline Often & $11(8.7)$ & $21(17.8)$ & $21(21.4)$ & \\
\hline \multicolumn{5}{|l|}{ Wash hands } \\
\hline No & $9(7.1)$ & $7(6.0)$ & $8(8.3)$ & 0.095 \\
\hline Sometimes & $34(27.0)$ & $50(42.7)$ & $38(39.6)$ & \\
\hline Always & $83(65.9)$ & $60(51.3)$ & $50(52.1)$ & \\
\hline \multicolumn{5}{|l|}{ Family size } \\
\hline$\leq 5$ & $96(76.2)$ & $95(80.5)$ & $66(67.4)$ & 0.079 \\
\hline$\geq 6$ & $30(23.8)$ & $23(19.5)$ & $32(32.6)$ & \\
\hline \multicolumn{5}{|l|}{ Share bedroom with: } \\
\hline No one & $34(27.2)$ & $27(23.3)$ & $17(17.7)$ & 0.134 \\
\hline 1 person & $62(49.6)$ & $66(56.9)$ & $47(49.0)$ & \\
\hline$\geq 2$ people & $29(23.2)$ & $23(19.8)$ & $32(33.3)$ & \\
\hline \multicolumn{5}{|l|}{ Type of bed } \\
\hline Wood & $65(52.0)$ & $66(56.4)$ & $50(51.0)$ & 0.548 \\
\hline Tatami* & $6(4.8)$ & $9(7.7)$ & $4(4.1)$ & \\
\hline Simmons $\dagger$ & $54(43.2)$ & $42(35.9)$ & $44(44.9)$ & \\
\hline \multicolumn{5}{|l|}{ House style } \\
\hline Single-family detached & $3(2.4)$ & $19(16.2)$ & $5(5.2)$ & 0.001 \\
\hline Apartment & $72(57.1)$ & $59(50.4)$ & $46(47.4)$ & \\
\hline Townhouse & $51(40.5)$ & $39(33.3)$ & $46(47.4)$ & \\
\hline
\end{tabular}

* Japanese straw matting.
$\dagger$ Local term for western-style bed with mattress and box spring.

TABLE 3

Crude odds ratios from univariate analyses and adjusted odds ratios from multivariate logistic regression analysis for factors potentially associated with pinworm infection in the case-control study in Taichung County and Kaohsiung City, Taiwan, combined

\begin{tabular}{|c|c|c|c|c|}
\hline \multirow[b]{2}{*}{ Variable } & \multirow[b]{2}{*}{$\begin{array}{l}\text { No. } \\
\text { controls }\end{array}$} & \multirow[b]{2}{*}{$\begin{array}{l}\text { No. } \\
\text { cases }\end{array}$} & \multicolumn{2}{|c|}{ Odds ratios } \\
\hline & & & $\begin{array}{c}\text { Crude (95\% } \\
\text { confidence interval) }\end{array}$ & $\begin{array}{c}\text { Adjusted (95\% } \\
\text { confidence interval) }\end{array}$ \\
\hline \multicolumn{5}{|l|}{ Sex } \\
\hline Female & 121 & 185 & 1.0 & 1.0 \\
\hline Male & 161 & 240 & $0.98(0.72-1.32)$ & $0.73(0.53-1.03)$ \\
\hline \multicolumn{5}{|l|}{ Age } \\
\hline$\geq 10$ years & 125 & 145 & 1.0 & 1.0 \\
\hline$\leq 9$ years & 159 & 286 & $1.55(1.14-2.11)$ & $1.44(1.04-2.00)$ \\
\hline \multicolumn{5}{|l|}{ Play on floor } \\
\hline Rarely & 178 & 167 & 1.0 & 1.0 \\
\hline Often & 105 & 261 & $2.65(1.94-3.61)$ & $2.52(1.80-3.51)$ \\
\hline \multicolumn{5}{|l|}{ Bite nails } \\
\hline No & 182 & 196 & 1.0 & 1.0 \\
\hline Yes & 101 & 234 & $2.15(1.58-2.93)$ & $2.08(1.50-2.88)$ \\
\hline \multicolumn{5}{|c|}{ Wash hands before meals } \\
\hline Yes & 171 & 199 & 1.0 & 1.0 \\
\hline Rarely & 113 & 232 & $1.76(1.30-2.39)$ & $1.71(1.23-2.37)$ \\
\hline \multicolumn{5}{|c|}{ House lived in } \\
\hline Apartment & 89 & 121 & 1.0 & 1.0 \\
\hline Other & 194 & 307 & $1.16(0.84-1.62)$ & $1.56(1.04-2.35)$ \\
\hline \multicolumn{5}{|c|}{ Residential area } \\
\hline Kaohsiung & 126 & 216 & 1.0 & 1.0 \\
\hline Taichung & 158 & 213 & $0.79(0.58-1.06)$ & $0.67(0.47-0.98)$ \\
\hline
\end{tabular}

blanket mass medication campaign with no screening is a great step in reducing high prevalence in a shorter period. A screening-medication program must be followed once the prevalence drops significantly.

Pinworm infection is regarded as the most common metazoan endoparasite in humans, but is also considered the least pathogenic of all human parasitic infections. ${ }^{10,11}$ Pruritis ani is the most striking symptom because the female worm may migrate to and deposit their eggs onto the perianal skin. Urinary tract infection is most common in girls with pinworm infection. Ok and others ${ }^{12}$ found $36.4 \%$ of girls with urinary tract infections had pinworm eggs by cellophane tape examination, whereas only $16.4 \%$ of the controls had the infection. Pinworm can also cause conditions such as postmenopausal bleeding, ${ }^{13,14}$ perianal granuloma, ${ }^{15}$ and hemorrhagic eosinophilic enterocolitis. ${ }^{16}$ Although mass screening campaigns and remediation for parasite infection may be costly, treatment of pinworm infection improves the quality of life for children. This parasitic infection should not be regarded as only a nuisance. The campaign for pinworm prevention and control for primary-school children received positive responses from the majority of the parents in Taiwan.

The chronological pattern of pinworm infection rate for first-graders entering the prevention program in each school year demonstrates that it may take 5-6 years to bring the 
infection rate from a level of $10 \%$ or higher down to a level of $1.0 \%$ or lower. This may translate into at least 16 followup screening examinations beginning with first-graders. Reinfection is probably an important factor associated with prolonged efforts in screening and treatment. Reinfection may increase the infection rate rebound to an additional $\geq 4 \%$ in all cohorts, when first-grade schoolchildren return to school after a summer break. It is unlikely that reinfection occurs at school because schools are closed during summer breaks.

On the basis of this case-control study, the home is a possible source of pinworm reinfection for children in Taiwan. A female adult pinworm may produce 10,000 fertilized, nonembryonated eggs that are expelled in the perianal area and distributed into the surrounding environment. ${ }^{11}$ It has been suspected in cross-sectional studies that dust-borne infection, indirect infection through clothing, bedding, or food, or direct infection by hand from anus to mouth is possible, particularly for children in rural areas with inadequate personal hygiene. ${ }^{4-7}$ Family socioeconomic status has been found to be significantly associated with pinworm infection. ${ }^{4}$ Chih and others ${ }^{7}$ also indicated children's habit of sucking fingers (OR, 2.01 for often versus never) and eating snacks (OR, 1.90 for often versus never) as significant risk factors for pinworm infection. The current case-control study has demonstrated that children who bite their fingernails or who do not wash their hands before meals are at higher risk for ingesting eggs from the environment. Geophagia may also occur, particularly after playing on the floor, with the risk being elevated to 2.5 times. Pinworm eggs may thus be transferred from hands to mouth.

Analyses of the chronological pattern for the entire population of primary-school children and the case-control study showed that the children's susceptibility to pinworm infection decreases with age. This difference in susceptibility is also in part due to children's daily behavior. Older children are less likely than younger children to play on the floor, to bite their nails, and to fail to wash their hands before meals.

This study adds further knowledge to the epidemiology and population-based control of intestinal parasites and pinworms, and provides a model of infection control among primary-school children in tropical areas. The strategies for prevention of intestinal helminth infection pursued in Taiwan can be adapted to developing programs in countries a endemic with parasites.

Acknowledgments: This study received a grant from the Taiwan Provincial Health Department and assistance from the Parasite Control Association.

Authors' addresses: John F. C. Sung, Sin Y. Wang, and Yun-Ju Lu, Institute of Environmental Health, National Taiwan University Col- lege of Public Health, 1 Jen Ai Road, Section 1, Taipei, Taiwan 100. Ruey S. Lin and Kuo C. Huang, Institute of Epidemiology, National Taiwan University College of Public Health, 1 Jen Ai Road, Section 1, Taipei, Taiwan 100.

Reprint requests: John F. C. Sung, Institute of Environmental Health, National Taiwan University College of Public Health, 1 Jen Ai Road, Section 1, Taipei, Taiwan 100, Telephone: 886-2-2312-3456 ext. 8461, Fax: 886-2-2394-8006 (e-mail: sung@ ha.mc.ntu.edu.tw).

\section{REFERENCES}

1. Akulongwe PF, Onwuliri CO, Titanji VP, Okwuosa VN, 1995. Prevalence of schistosomiasis and other intestinal helminth infections among senior primary school children in Makenene sub-division, Cameroon. J Helminthol 69: 103-105.

2. Glickman LT, Camara AO, Glickman NW, McCabe GP, 1999. Nematode intestinal parasites of children in rural Guinea, Africa: prevalence and relationship to geophagia. Int J Epidemiol 28: $169-174$.

3. Parasite Control Association, 1997. Statistics on Parasite Infection, 1976-1995. Taipei: Taiwan Parasite Control Association.

4. Chang JH, Huang WH, Chen ER, Hu SC, 1990. Survey of Enterobius vermicularis infection among school children in Tainan City. Kaohsiung J Med Sci 6: 587-593.

5. Chyr HW, Chen JG, 1993. Comparison of Enterobius vermicularis infection among preschool children in Ta-Liao District of Kaohsiung County with that in Kaohsiung City. Kaohsiung J Med Sci 9: 418-427.

6. Pai HH, Chen ER, 1988. A study of multiple factors related to Enterobius infection among pre-school children. Kaohsiung $J$ Med Sci 4: 217-230.

7. Chih HW, Lin HS, Tang KS, Cheu SC, 1996. A study of multiple factors related to Enterobius vermicularis infection among pre-school children in Ta-Liao district of Kaohsiung County. Kaohsiung J Med Sci 12: 538-543.

8. Department of Health, 1986 and 1998. Health and Vital Statistics, 1986 and 1998. Taipei: ROC Department of Health.

9. Council for Economic Planning and Development, Republic of China, 1997. Taiwan Statistical Data Book, 1997. Taipei: Council for Economic Planning and Development.

10. Garcia LS, Bruckner DA, 1989. Diagnostic Medical Parasitology. New York: Elsevier.

11. Despommier DD, Gwadz RW, Hotez PJ, 1995. Parasitic Diseases. Third edition. New York: Springer Verlag.

12. Ok UZ, Ertan P, Limoncu E, Ece A, Ozbakkaloglu B, 1999. Relationship between pinworm and urinary tract infections in young girls. APMIS 107: 474-476.

13. Surmont I, Liu LX, 1995. Enteritis, eosinophilia, and Enterobius vermicularis. Lancet 346: 1167.

14. Al-Rufaie HK, Rix GH, Perez Clemente MP, al-Shawaf T, 1998. Pinworms and postmenopausal bleeding. J Clin Pathol 51: 401-402.

15. Avolio L, Avoltini V, Ceffa F, Bragheri R, 1998. Perianal granuloma caused by Enterobius vermicularis: report of a new observation and review of the literature. J Pediatr 132: 10551056.

16. Liu LX, Chi J, Upton M, Ash LR, 1995. Eosinophilic colitis associated with larvae of the pinworm Enterobius vermicularis. Lancet 346: 410-412. 